\title{
Pulmonary tuberculosis in a male with silicosis
}

The authors declare no financial disclosure

\begin{abstract}
Silicosis, a preventable occupational lung disease, is associated with various diseases, including tuberculosis (TB). There is an increased lifelong risk for lung tuberculosis even if exposure to silica dust ceases. That association contributes in a large extent to very high rates of tuberculosis in countries with poor TB and silica exposure control. We report a case of a male with a prior diagnosis of silicosis who was diagnosed with and treated for TB. Anti-tuberculous treatment was extended due to a positive sputum smear in the six ${ }^{\text {th }}$ month of treatment. However, the culture of the sputum was negative. This case report highlights the value of thorough screening for tuberculosis in silicotic patients. The clinical management of these patients may be challenging due to frequent overlapping radiological features and uncertain results of TB treatment.
\end{abstract}

Key words: tuberculosis, silicosis, exposure, occupational, dust

\section{Case report}

Silicosis is a fibrotic lung disease resulting from exposure to silica dust. Worldwide, it is regarded as the most frequent occupational lung disorder. Although it is relatively rare in the developed countries, it is still fairly common in countries with poor occupational control such as the Republic of South Africa. It has been recognized for years that there is a relationship between tuberculosis (TB) and silicosis. In fact, it has been reported that $25-30 \%$ of silicotic patients develop TB [1]. That adverse association is very common among workers in South Africa's gold mining industry. The incidence of TB in South Africa is as high as 2707 per 100,000 in men with silicosis [1].

Given the fact that there is such a strong relationship between TB and silicosis, we report a case of a male with predisposing silicosis and subsequent pulmonary TB.

A 58-year-old non-smoking male was admitted to the tuberculosis ward with a history of a
2 monthlong moderate cough with mucoid sputum production, a monthlong fever (axillar temperature up to $39^{\circ} \mathrm{C}$ ), night sweats, anorexia and loss of weight (8 kilos). He did not report any chest pain and hemoptysis. As an outpatient, he was treated with clarithromycin and ciprofloxacilin with no significant clinical improvement. His medical history was remarkable for silicosis. It was diagnosed 15 years ago and since then he was regularly seen in the respiratory clinic. He used to work in an aluminium foundry for 17 years. Consequently, the long exposure to silica dust was recognized and the state benefit for silicosis as occupational disease was granted. He stopped smoking 30 years ago and rarely drank alcohol. He had no contact with a confirmed TB source. On examination, he was excessively kyphotic in cervical and thoracic region of the spine, visibly underweight (body-mass-index [BMI] $17 \mathrm{~kg} /$ $\mathrm{m}^{2}$ ), slightly hypertensive (BP 150/90 mm Hg) and tachycardic (HR 100/min) with clear breath sounds on auscultation. On admission, his chest X-ray (CXR) (Fig. 1 A, B) showed multiple small

Address for correspondence: dr n. med. Marcin Skowroński, Wielkopolskie Centrum Pulmonologii i Torakochirurgii, Ludwikowo k/Poznania, Mosina 62-050

e-mail: marcinas@gazeta.pl, tel.: +48607669 013, fax: +48618132879.

DOI: 10.5603/ARM.2018.0019

Received: 20.02.2018

Copyright (C) 2018 PTChP

ISSN 2451-4934 

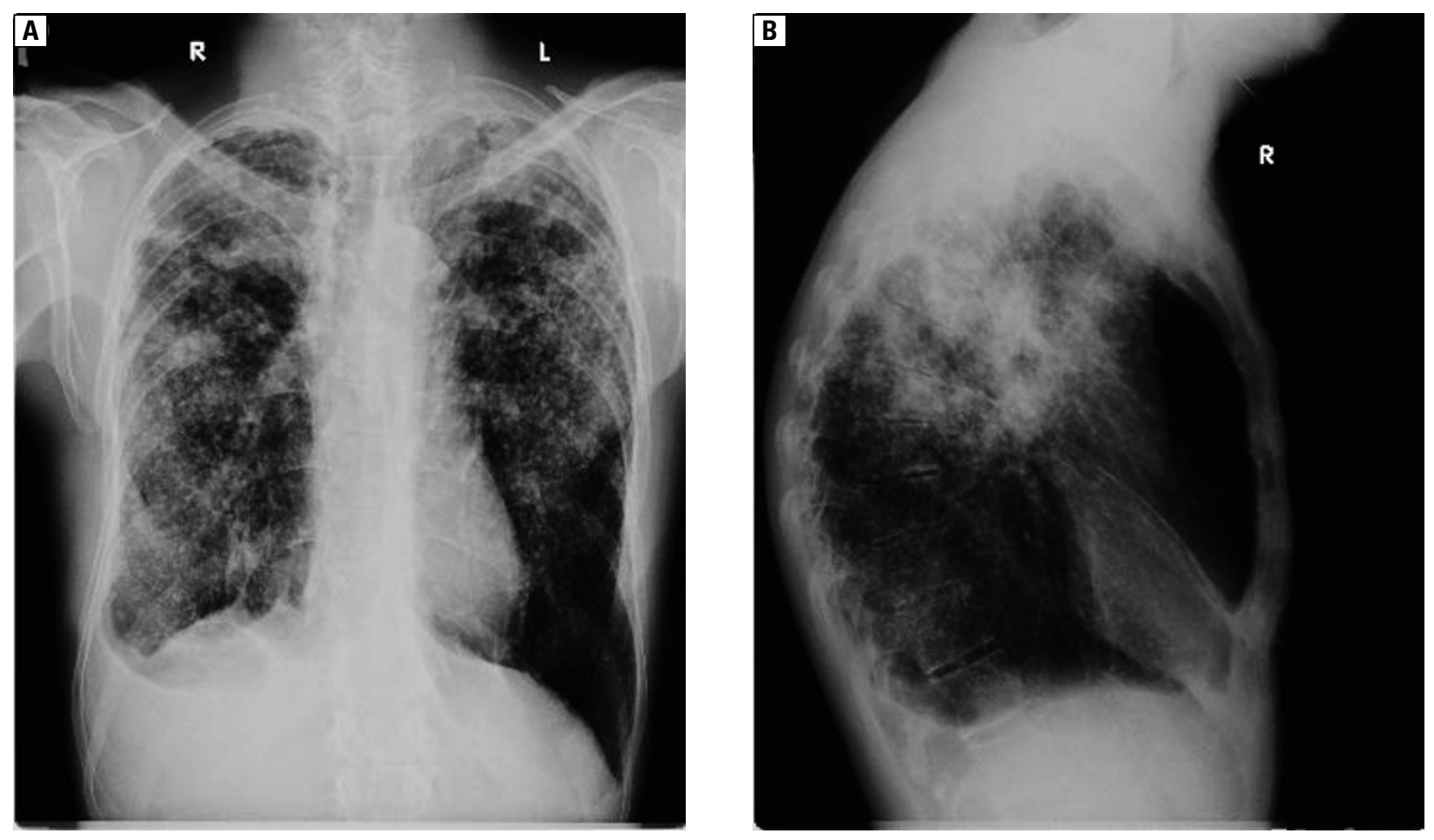

Figure 1 A, B. Multiple confluent small nodules and masses in the upper right and left zones, consolidation in the middle right zone and small right pleural effusion

nodules in the upper right and left zones, consolidation in the middle right zone and small right pleural effusion. Inflammatory markers were moderately raised (erythrocytes sedimentation rate $-68 \mathrm{~mm} / \mathrm{h}$, C-reactive protein $35 \mathrm{mg} / \mathrm{L}$ ). His sputum smear was positive for acid-fast bacilli (AFB) $(+++)$. Mycobacterium tuberculosis complex was confirmed with GeneXpert MTB/RIF test. The strain was deemed rifampicin sensitive as no mutation was found in the rpoB gene. Anti-tuberculous treatment (ATT) was started with rifampicin (R), isoniazid (H), ethambutol (E) and pyrazinamide $(\mathrm{Z})$ and piridoxine. Consequently, after 9 days, Mycobacterium tuberculosis was cultured and identified with MGIT (Mycobacteria Growth Indicator Tube). Drug susceptibility testing (DST) showed sensitivity to all first-line drugs. At the beginning, the tolerance of ATT was poor with loss of appetite and an increase in liver function tests (LFT). The patient improved gradually with resolution of cough and weight gain. His sputum samples were negative for AFB after 2 months of ATT. Subsequently, after 2 months of in-hospital treatment he was discharged and treated as an outpatient with HR as a continuation phase for the next 4 months. He was admitted again in the $6^{\text {th }}$ month of ATT due to the positive sputum smear $(\mathrm{AFB}+)$. On admission, he was well and reported nonfrequent cough and an increase in weight. The following sputum specimens as well as bronchial washing were smear negative for AFB. ATT was continued with good tolerance while the cultures on liquid medium (MGIT) were monitored. Computed tomography (CT) of the chest revealed scattered calcified nodules in various sizes, irregular fibrotic mass with a central cavity in the right upper lobe as well as a thick-walled cavity measuring $36 \times 46$ $\mathrm{mm}$ in the left upper lobe. CT also showed hilar calcified lymph nodes. CXR discovered further resolution in comparison with that previous one on admission (Fig. 2). After culture conversion (no positive results of cultures) and extension of ATT to 9 months, the patient was discharged with a close follow-up in a respiratory clinic. Thus, the positive AFB smear in the $6^{\text {th }}$ month of ATT was considered a nonviable strain due to the negative culture.

\section{Discussion}

The association between silicosis and TB has been well established. Recent findings show that an exposure to silica in patients without silicosis may predispose to TB [2]. That risk seems to be lifelong even if exposure ceases. Moreover, the frequency of TB was 3 to 4 times higher in those patients with heavy and longer than 


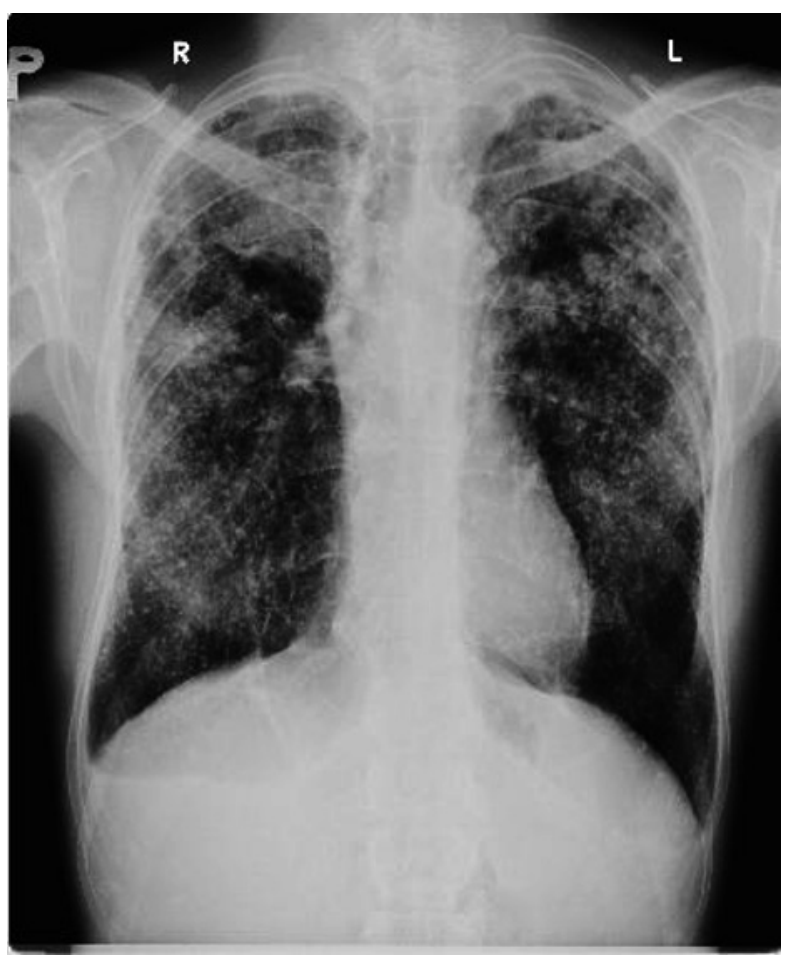

Figure 2. Partial resolution of consolidation and pleural effusion after 6 months of ATT

15 years exposure. This relationship was confirmed in a large prospective cohort study of 1153 gold miners with and without silicosis [3]. The relative risk of TB was 2.8 (95\% CI, 1.9-4.1) in silicotic men. That risk was elevated to the same extent for extrapulmonary TB.

The mechanisms underlying that association are not clear. It was suggested that inhaled silica modifies the immune reponse of the lung, impairs the macrophages function and causes their apoptosis. This finding can be confirmed by the observation that heavier exposure may result in higher TB incidence [3]. In addition, surfactant protein $\mathrm{A}$ is elevated in the bronchoalveolar lavage (BAL) fluid of silicotic patients. Its excess seems to be linked with increased TB risk [3]. Finally, the TB bacilli can remain dormant for a long time within the silicotic nodules [3]. Direct impairment of macrophages and poor drug penetration into these nodules can be responsible for a subsequent treatment failure and high relapse rate in these patients.

Of note, silicosis and previous TB treatment are also the recognized risk factors for non-tuberculous mycobacteria (NTM) infections (the mean odds ratio (OR) 12.6 and 3.6, respectively [4]. Mycobacterium kansasii is found to be the most prevalent species.

In a study of agate stone workers in India, OR for an association between silicosis and TB was
2.75 [5]. Moreover, the latter was a significant contributor to mortality. Likewise, the risk of TB was elevated nearly three times in silicotic gold miners in comparison with the subjects with no radiographic evidence of silicosis [3]. In patients with no silicosis, the TB risk was found to be directly related to the cumulative degree of exposure. The diagnosis was made on average 7.6 years after the end of exposure. This indicates that after the removal of silica source and regardless of silicosis, this population is still at a lifelong risk of TB. The large exposure to crystalline silica is also a major factor in extraordinary TB incidence in one of the regions of Portugal [6]. Likewise, silica exposure was prevalent among TB patients (OR: 3.39, 95\% CI, 2.6-4.3) [2]. The incidence of TB was higher among patients with long exposure ( $>$ 15 years), advanced age, lower level of education, male gender and smoking.

One of the risk factors for TB among silicotic patients is smoking (adjusted hazard factor 1.96, 95\% CI, 1.1-3.3) [7]. It increases both risk of latent TB infection and later progression to the disease. Also, there was observed a clear dose-response relationship between the number of cigarettes smoked a day and a number of active TB cases. On the other hand, cigarette pack-year was a weak risk factor for the development of TB [8]. Significant risk factors for TB among silicotic patients were as follows: lack of anti-tuberculous treatment (ATT) prior to silicosis diagnosis, progressive massive fibrosis (PMF), small opacities exceeding $1.5 \mathrm{~mm}$ and caisson work [8]. The combination of PMF and an absence of ATT before silicosis identified the group with the highest TB risk.

There is evidence that TB may affect negatively the clinical course of simple pneumoconiosis. In a prospective observation, TB was found to be a main risk factor for premature death (relative risk (RR) 2.0, p < 0.01) and the development of progressive massive fibrosis (RR 7.0, $p<0.01$ )[9]. It was also found that there was a signicantly elevated mortality due to TB among silica exposed workers (whetstone cutters) [10]. In that large analysis, in the cause of death of 99 cases among cohort of 200 males from 1955 to 1995, there were 10 TB cases (of which 9 cases had silicosis). In contrast, in the United States, there was a decline in silicosis-respiratory TB mortality (from 239 per year during 1968-1972 to 1.2 per year during 2002-2006) [11]. This drop probably reflects both prevention and better control measures of both diseases.

The diagnosis of active TB superimposed on silicosis can be clinically challenging. The 
principal imaging findings consistent with TB are thick-walled cavities, consolidations, tree-in-bud pattern, nodular image asymmetry and rapid disease progression [12]. Of these, cavitation is the strongest indicator of likely silicotuberculosis. In a patient with a suggestive occupation history, it is reasonable to evaluate old serial X-rays to search for any progression of the disease. Since both diseases have a similar mode of presentation, there is a chance of their concurrent existence. However, the silica exposure may alter the radiological resolution of miliary TB. As a consequence, there can be more pronounced fibrotic response to TB in silicotic patients, which leads to a delayed and less complete improvement of milliary TB [13]. Of note, there is evidence of long-term respiratory consequences of treated TB in silica-dust exposed males. In South African miners, TB was associated with a decline in pulmonary function tests (a drop of $40 \mathrm{ml} /$ year in forced expiratory volume in 1 second $\left(\mathrm{FEV}_{1}\right)$ and $43 \mathrm{ml} /$ year in forced vital capacity (FVC) [14]. In addition, TB cases had more than double chances of breathlessness at follow-up. This loss of lung function was greater among those with more severe respiratory symptoms and later clinical presentation of TB.

According to World Health Organization (WHO) guidelines, patients with silicosis should be screened with interferon-gamma release assays (IGRA) or tuberculin skin test for latent tuberculosis infection (LTBI) [15]. Both testing and preventive treatment of silicosis patients with LTBI are recommended in high- or middle-income countries with a low TB incidence. The rifampicin or isoniazid monotherapy with the least hepatotoxity might be the first choice in the preventive therapy. The other treatment option might be combined isoniazid and rifampicin regimen. Of interest, tuberculin-positive patients with silicosis have a 30 times higher risk of developing TB [11]. However, there is some doubt concerning the benefit of IGRA in the clinical management of patients with silicosis. There is evidence of persistent of interferon-gamma response among German miners with no recent TB exposure [16]. The predictors of positive IGRA results were age $>80$ years, foreign place of birth and radiologically healed prior TB but not silicosis, and smoking. No active TB case during a follow-up was detected. Hence, the clinical value of current IGRAs among patients exposed to silica remains uncertain. That doubt is related to the limited efficacy of preventive treatment in patients with TB and silicosis as well as the risk of drug-induced hepatitis. In a double-blind randomized study, the different regimens of chemoprophylaxis in silicotic patients reduced TB development, though the proportion of active TB cases remained substantial [17]. On the other hand, a comprehensive approach is required to prevent silica associated diseases, including better dust exposure control. The clinical management of these diseases should be consistent with a Global Elimination of Silicosis Campaign organized by the International Labour Organization (ILO) and WHO [18].

In conclusion, patients with a history of exposure to silica or silicosis need a regular and long-term surveillance as the TB risk in this group is significantly elevated - even threefold. The risk is found to be lifelong even if exposure ceases. Like in our patient, any typical of TB but not specific features in the silicotic subject such as persistent cough, weight loss, fever should be thoroughly examined to exclude TB. In addition, any radiological findings such as asymmetric nodules, rapid progression or cavitation should raise concern. Treatment of TB in these patients may be extended as sputum samples may remain longer smear-positive . Finally, both silicosis and silicotuberculosis patients should be under close and regular follow-up in order to detect early any new TB case or relapse.

\section{Conflict of interest}

The authors declare no conflict of interest.

\section{References:}

1. Cowie RL. The epidemiology of tuberculosis in gold miners with silicosis. Am J Respir Crit Care Med. 1994; 150(5 Pt 1): 1460-1462, doi: 10.1164/ajrccm.150.5.7952577, indexed in Pubmed: 7952577.

2. Yarahmadi A, Zahmatkesh MM, Ghaffari M, et al. Correlation between silica exposure and risk of tuberculosis in lorestan province of Iran. Tanaffos. 2013; 12(2): 34-40, indexed in Pubmed: 25191460.

3. Hnizdo E, Murray J. Risk of pulmonary tuberculosis relative to silicosis and exposure to silica dust in South African gold miners. Occup Environ Med. 1998; 55(7): 496-502, indexed in Pubmed: 9816385.

4. Murray J, Sonnenberg P, Shearer S, et al. Risk factors for pulmonary disease due to culture-positive M. tuberculosis or nontuberculous mycobacteria in South African gold miners. Eur Respir J. 2000; 15(2): 291-296, indexed in Pubmed: 10706494.

5. Chaudhury N, Paliwal R, Phatak A. Co-morbidities among silicotics at Shakarpur: A follow up study. Lung India. 2012; 29(1): 6-10, doi: 10.4103/0970-2113.92348, indexed in Pubmed: 22345906.

6. Coutinho D, Sousa P, Oliveira O, et al. Can silicosis explain it all? Rev Port Pneumol (2006). 2016; 22(6): 358-360, doi: 10.1016/j.rppnen.2016.07.004, indexed in Pubmed: 27650457.

7. Leung CC, Yam WC, Yew WW, et al. Determinants of spirometric abnormalities among silicotic patients in Hong Kong. Occup Med (Lond). 2005; 55(6): 490-493, doi: 10.1093/occmed/ kqi107, indexed in Pubmed: 16140838.

8. Chang KC, Leung CC, Tam CM. Tuberculosis risk factors in a silicotic cohort in Hong Kong. Int J Tuberc Lung Dis. 2001; 5(2): 177-184, indexed in Pubmed: 11258512. 
9. Yi Q, Zhang Z. The survival analyses of 2738 patients with simple pneumoconiosis. Occup Environ Med. 1996; 53(2): 129-135, indexed in Pubmed: 8777450.

10. Ogawa S, Imai H, Ikeda M. Mortality due to silico-tuberculosis and lung cancer among 200 whetstone cutters. Ind Health. 2003; 41(3): 231-235, indexed in Pubmed: 12916753.

11. Nasrullah M, Mazurek JM, Wood JM, et al. Silicosis mortality with respiratory tuberculosis in the United States, 19682006. Am J Epidemiol. 2011; 174(7): 839-848, doi: 10.1093/aje/ kwr159, indexed in Pubmed: 21828370.

12. Chong S, Lee KS, Chung MJ, et al. Pneumoconiosis: comparison of imaging and pathologic findings. Radiographics. 2006; 26(1): 59-77, doi: 10.1148/rg.261055070, indexed in Pubmed: 16418244.

13. Charalambous S, Churchyard GJ, Murray J, et al. Persistent radiological changes following miliary tuberculosis in miners exposed to silica dust. Int J Tuberc Lung Dis. 2001; 5(11): 1044-1050, indexed in Pubmed: 11716341.

14. Ross J, Ehrlich RI, Hnizdo E, et al. Excess lung function decline in gold miners following pulmonary tuberculosis. Thorax.
2010; 65(11): 1010-1015, doi: 10.1136/thx.2009.129999, indexed in Pubmed: 20871124.

15. Getahun H, Matteelli A, Abubakar I, et al. Management of latent Mycobacterium tuberculosis infection: WHO guidelines for low tuberculosis burden countries. Eur Respir J. 2015; 46(6): 1563-1576, doi: 10.1183/13993003.01245-2015, indexed in Pubmed: 26405286.

16. Ringshausen FC, Nienhaus A, Schablon A, et al. Frequent detection of latent tuberculosis infection among aged underground hard coal miners in the absence of recent tuberculosis exposure. PLoS One. 2013; 8(12): e82005, doi: 10.1371/journal. pone.0082005, indexed in Pubmed: 24312620.

17. A double-blind placebo-controlled clinical trial of three antituberculosis chemoprophylaxis regimens in patients with silicosis in Hong Kong. Hong Kong Chest Service/Tuberculosis Research Centre, Madras/British Medical Research Council. Am Rev Respir Dis. 1992; 145(1): 36-41, doi: 10.1164/ ajrccm/145.1.36, indexed in Pubmed: 1731596.

18. Fedotov I. Global elimination of silicosis: the ILO/WHO international programme. Asian-Pacific Newsletter on Occupational Health and Safety. 1997; 4(2). 